\title{
Laparoscopic Appendectomy
}

National Cancer Institute

\section{Source}

National Cancer Institute. Laparoscopic Appendectomy. NCI Thesaurus. Code C51893.

Surgical removal of the vermiform appendix, using a laparoscope. 